\title{
Physical Fatigue Comparison of Eco-Driving and Normal Driving*
}

\author{
Shigeyuki YAMABE**, Rencheng ZHENG**, Kimihiko NAKANO** \\ and Yoshihiro SUDA** \\ ** Institute of Industrial Science, The University of Tokyo \\ 4-6-1 Komaba, Meguro-ku, Tokyo, Japan \\ E-mail: yamabe@iis.u-tokyo.ac.jp
}

\begin{abstract}
Nowadays, eco-driving as a series of driving behaviors is proposed to reduce $\mathrm{CO}_{2}$ discharge of vehicle and realize safe driving. The eco-driving behaviors are mainly about accelerator and brake works that are operated by leg's motions of driver; however, there are few studies to explain physical fatigue resulted by leg`s motions for eco-driving behaviors. In the paper, eco-driving experiment was conducted in the modes of normal driving, eco-driving with instruction, and eco-driving with eco-indicator. The simulated driving experiment was realized by a universal driving simulator system, and surface electromyography of leg muscle of 10 subjects was measured to clarify physical fatigue of driver in different driving modes. The experiment indicates that fuel economy in eco-driving with eco-indicator is higher than other driving modes, and muscle activities are lowest by signal analysis of the surface electromyography. The result also suggests that eco-indicator is effective assistant system to help driver realize eco-driving as well as reduce physical burden of driver.
\end{abstract}

Key words: Automobile, Human Interface, Driving Simulator, Driver Assist, Eco-Drive Behaviors, Physical Fatigue

\section{Introduction}

To prevent global warming, international agreement of Kyoto Protocol was concluded in 1997, government of Japan announced that $25 \%$ reduction of $\mathrm{CO}_{2}$ discharge would be as target compared with $1990 . \mathrm{CO}_{2}$ discharge by transportation sector is about $23 \%$ of total $\mathrm{CO}_{2}$ discharge in all over the world, and reduction of $\mathrm{CO}_{2}$ discharge in transportation sector becomes urgent. Generally, fuel consumption can be cut down by proper driving behaviors and improvement of vehicle performance.

Dependent on above demands, eco-driving is proposed to improve fuel economy for reduction of $\mathrm{CO}_{2}$ discharge. Eco-driving has an immediate and significant effect on improving vehicle's fuel economy; specifically, eco-driving can improve fuel consumption about $10-30 \%$, which is greatly potential for reduction of $\mathrm{CO}_{2}$ emissions ${ }^{(1)}$. In Japan, the eco-driving is generally a driving method about driver behaviors such as acceleration at start, preservation of steady speed, active use of engine brake. The eco-driving techniques in Europe, especially in German, differ from the eco-driving techniques in Japan, and main content is to accelerate early to the fuel efficient range. The eco-driving is a promising method to reduce $\mathrm{CO}_{2}$ discharge, and several related studies had been reported. John et al. ${ }^{(2)}$ suggested greenhouse gas reduction including the eco-driving by using intelligent transportation systems (ITS) technology. Hiraoka et al. quantitatively evaluated the fuel 
consumption of eco-driving by comparison of Japanese and Germany eco-driving ${ }^{(3)}$. Moreover, related techniques of eco-driving were also developed to better eco-driving for driver ${ }^{(4)}$.

However, most of former studies about eco-driving concentrated on fuel consumption, and few considered human factors in eco-driving system. The human factors include driving behaviors, driving habits, and driver burden ${ }^{(5)}$. Especially, pedal operations of the eco-driving is relatively complicated, such as acceleration smoothly at start, few acceleration and deceleration, and early pedal release, which operations may lead to driver fatigue. In a word, some problem about influence of eco-driving on driver is not clear until now. About eco-driving manners of Japan, the eco-driving behaviors are mainly about accelerator and brake works that are operated by leg`s motions of driver. By experimental studies, the study is try to use surface electromyography (sEMG) signals for explaining physical fatigue resulted by leg's motions for eco-driving behaviors.

In the paper, traffic environment of simulated driving is introduced to prepare driving experiment. Then, sEMG signals of leg muscles of 10 subjects were measured to deeply understand driver behaviors in eco-driving. Based on measured signal of sEMG, physical fatigue of subjects under different driving modes were analyzed and discussed.

\section{Methodology}

\subsection{Eco-driving Behaviors}

Eco-driving behaviors are advocated in Japan, and Eco-driving Ten Points had been established as follows ${ }^{(6)}$,

(1) accelerate gently at start,

(2) keep steady speed,

(3) accelerate off as early as possible,

(4) control using of air conditioner,

(5) cut down engine idling,

(6) limit engine warm-up,

(7) actively use road and traffic information,

(8) check air pressure of tire regularly,

(9) reduce loading in car,

(10) obey parking regulations to reduce traffic jams.

The points of (1), (2), and (3) are used to improve fuel economy by driver operations on accelerator and brake works. The points of (4), (5), (6), (8), and (9) are the methods that correctly use equipments to reduce fuel consumption. The point (7) and (10) is about actively using read and traffic information to achieve destination early, and obey parking regulations to reduce traffic jams for reduction of fuel consumption. In a word, the ten points is greatly helpful to reduce fuel consumption of vehicle by everyday driving behaviors. This paper pays attention to points of (1), (2), and (3), which has a direct effect on the mileage by adjusting self- driving behaviors.

\subsection{Driving Simulator}

The eco-driving experiment was accomplished by a universal driving simulator (DS) in The University of Tokyo. The Fig. 1 shows the main constructions of the DS. Dynamic motions of vehicle are simulated by a moving platform of 6 degrees of freedom, and a turntable mechanism which improves turning performance of the $\mathrm{DS}^{(7)}$. Eight screens are equipped around the DS, and traffic vision with 360 degrees field is projected by using eight projectors. Additionally, the screens for side-view mirrors are also prepared, and therefore driver can drive in a driving environment that is closed to real environment. 


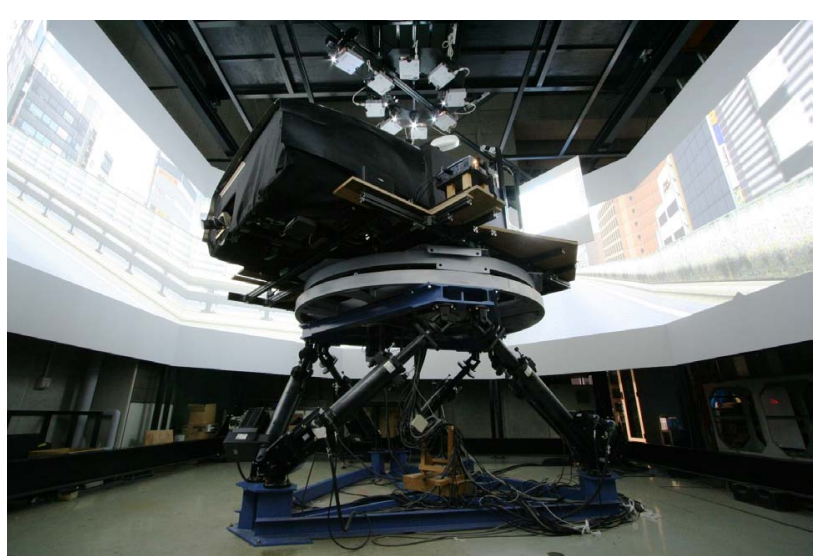

Fig. 1 Universal driving simulator

\subsection{Traffic Scenario}

A traffic layout of city area was designed as experimental scenario in the DS traffic system in Fig. 2. The course of the traffic layout is $3.4 \mathrm{~km}$, and driver should turn right two times and turn left two times, respectively. From start point in Fig. 2 (a), six traffic signals are set in different places of the scenario, the traffic signals change by the same timing. As is shown in Fig. 2 (b), bold line in Fig. 2 (a) is three lanes for each road, and thin line in Fig. 2 (a) is two lanes for each road. The driver could choose a favorite traffic lane, but wasteful route is prohibited. To investigate preliminary driving behaviors, other vehicles are not enclosed in the scenario, since subject maybe couldn't realize eco-driving by cooperation with other vehicles. In the experiment, the driving time is more than 30 minutes, and driving speed of $60 \mathrm{~km} / \mathrm{h}$ is required as a target. Full distance is about $24 \mathrm{~km}$, since the running speed is different for subjects.

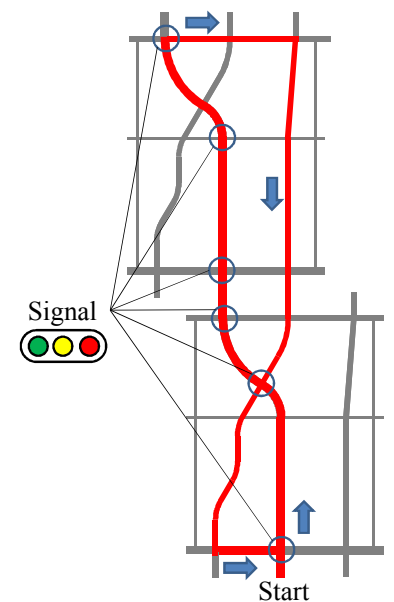

(a) Traffic layout

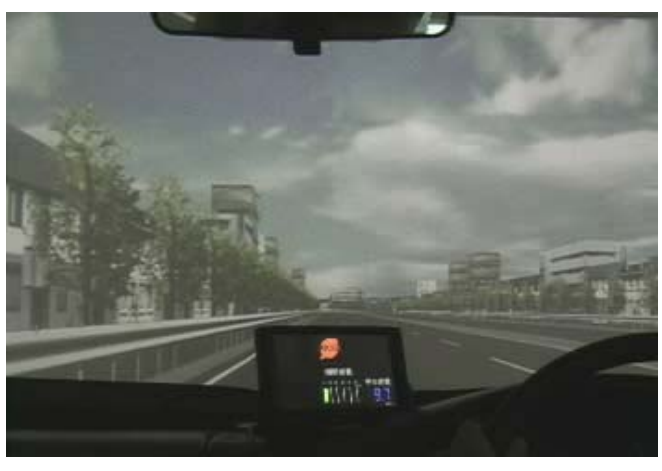

(b) Traffic environment

Fig. 2 Experimental scenario

The driving experiments were conducted in three driving modes in same time period of separate days. In the first day, a subject is asked to perform normal driving according to traffic rules in the scenario. And in the second day, the same subject received educations about Eco-driving Ten Points, and is asked to try to realize the eco-driving behaviors of (1), (2), and (3), which are about accelerating gently at start, keeping steady speed, and accelerating off as early as possible. In the third day, the same subject drives with assistant eco-driving equipment, which is an eco-indicator to provide real-time fuel economy. During the driving experiments, subject is also required to try to realize the eco-driving behaviors of (1), (2), and (3). 
During the driving experiments, signals of surface Electromyography (sEMG) of leg muscles of the driver were measured in following two periods,

[I] One lap after passing the first signal from the starting point of Fig. 2,

[II] One lap from the same point as [I] after 30 minutes of continuous driving.

\subsection{Eco-indicator}

For further analysis of fuel economy in eco-driving, a human machine interface as an eco-indicator was equipped into the DS system. By the eco-indicator, instantaneous fuel economy, average fuel economy, and eco-marks could be displayed in real-time feedback setting. The real-time data displayed in the monitor of eco-indicator is used to help driver to choose proper driving behaviors to reduce fuel consumption, and the data is also applied on learning of effectiveness of the eco-driving. The eco-indicator is shown in Fig. 3.

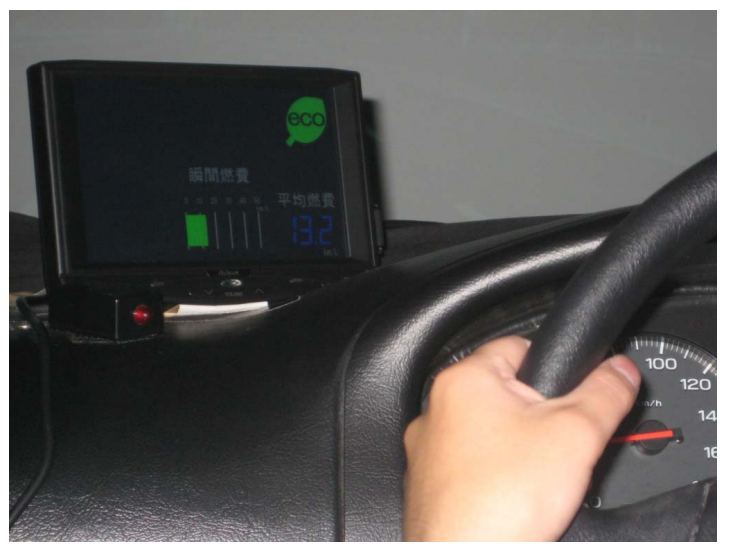

Fig. 3 Eco-indicator

There are three versions of the eco-marks in the eco-indicator system. First version is a brown eco-leaf, which is shown in the left of Fig. 3, and the average fuel economy is shown by a dead liked leaf in $7.0-10.0 \mathrm{~km} / 1$, and the average fuel economy is shown by a yellow leaf in $10.0-13.0 \mathrm{~km} / \mathrm{l}$, the average fuel economy is shown by a green leaf in 13.0 $20.0 \mathrm{~km} / 1$ (in Fig. 3). The eco-mark would be disappeared when the average fuel economy is over $20 \mathrm{~km} / \mathrm{l}$. The driver should improve his driving behaviors through observe the fuel economy in the eco-indicator. Moreover, the instantaneous fuel economy is also displayed by a real-time varying bar label, and the average fuel consumption is displayed by real-time values.

A modal emission model is used to calculate the fuel economy, and the model is presented by equations (1) and $(2)^{(8)}$.

$$
\begin{gathered}
\mathrm{FC}(t)=k_{\text {idle }} \cdot N_{\text {idle }} \cdot D, \quad \cdots \text { for } P=0 \\
\mathrm{FC}(t)=\lambda(t)\left(k(t) \cdot \frac{N(t)}{60} \cdot D+\frac{P_{\text {engine }}}{\eta_{\text {engine }}}\right) \cdot \frac{1}{H}, \cdots \text { for } P>0 \\
k(t)=k_{0}\left[1+\left(\frac{N(t)}{60}-33\right)^{2} \cdot 10^{-4}\right], \quad k_{0}=0.2
\end{gathered}
$$

When $P$ is engine power, the vehicle is stopping in $P=0$, and fuel economy $\mathrm{FC}[\mathrm{g} / \mathrm{s}]$ is used only idle the engines. $k$ expresses the engine individual difference, $N$ is engine speed, and $D$ is displacement. When $P>0, \mathrm{FC}$ is calculated by equation (2). $\lambda$ is air-fuel ratio. $P_{\text {engine }}$ and $\eta_{\text {engine }}$ show the loss of the engine and the engine efficiency. $P_{\text {engine }}$ includes transmission efficiency of the engine. $H$ is rotation frequency of the engine. This model can set a detailed variable from in-vehicle experiment. In our study, the model is used to match the DS. 
The instantaneous fuel economy is displayed on every second in eco-indicator. The average fuel economy is mean value of fuel economy for $100 \mathrm{~m}$. Furthermore, when a vehicle drives over a new $20 \mathrm{~m}$, the data about the oldest $20 \mathrm{~m}$ will be discarded, and the data about the new $20 \mathrm{~m}$ will be collected to the model to calculate the average fuel economy.

\section{5. sEMG Measuring}

During the eco-driving, the subject are asked to realize eco-driving points of (1), (2), and (3), which is about accelerate gently at start, keep steady speed, and accelerate off as early as possible. The three eco-driving points are about accelerator and brake works, which are accomplished by operations of right lower limb of driver. To observe physical condition of driver, sEMG signals of right leg were measured in the eco-driving experiment.

10 healthy males from 20 to 60 years old cooperated with the eco-driving experiments, and all of them hold common driving certificate of Japan. Before the sEMG measuring experiments, agreements were signed with 10 subjects dependent on ethical rules ${ }^{(9)}$.

Accelerator and brake works are mainly operated by muscle of shank. Therefore, sEMG signals of four muscles were measured including (1) tibialis anterior muscle, (2) lateral gastrocnemius muscle, (3) peroneus longus muscle, and (4) medialis gastrocnemius muscle. The measuring positions of the four muscle's sEMG are presented in Fig.4, measuring position of medialis gastrocnemius muscle is on opposite of lateral gastrocnemius muscle. The measuring positions are dependent on anatomical guide of surface electromypgraphy ${ }^{(10,11)}$. During the eco-driving experiment, active electrodes (Polymate AP1132, Teac Company) are applied for measurement of sEMG signals.

The tibialis anterior muscle is mainly activated when ankle is on flexion and extension motions $^{(12)}$. By measured sEMG signals in the eco-driving experiment, it is validated that accelerator and brake works are mainly completed by tibialis anterior muscle. Additionally, sEMG signal of tibialis anterior muscle is easy to measure and processing. Therefore, the paper is only discussed the sEMG signal of tibialis anterior muscle.



Fig.4 sEMG positions

\subsection{Signal Processing}

By measuring sEMG signal, the study aims at analysis of physical condition by observing muscular fatigue of leg, which results from driving behavior in designed driving experiments. When muscle gets tired and stretching exercise decreases, myoelectric potential increases for using more muscular strength as much as it's decreased. The phenomenon could be applied t explain muscular fatigue.

Following the muscular fatigue, the amplitude of EMG signal is being higher, and frequency of EMG signal is beding lower wave ${ }^{(13,14)}$. Therefore, root mean square (RMS) of EMG signal is commonly used to describe the signal amplitude content of the recorded sEMG signal, which is related with muscular fatigue, respectively ${ }^{(10)}$. To indicate muscular 
fatigue of leg, signal processing of sEMG signal is used by following steps,

(1) collect the raw signal of sEMG,

(2) rectify the raw signal of sEMG,

(3) smooth the sEMG data by a five-point moving average,

(4) normalize against the sEMG data by a five-point moving average of sEMG during maximum voluntary contraction (MVC) task,

(5) calculate muscular fatigue intensity using RMS value of the normalized signals.

The final RMS value is applied to indicate muscular fatigue intensity, and signal processing is shown by diagram procedure in Fig. 5 . When RMS value is used to indicate muscular fatigue, it is means that muscle activities and muscle fatigue is relatively high while the RMS value is high. For normalization of the measured EMG signals, sEMG signals of maximum voluntary contraction (MVC) for subject were measured before the driving experiment in the study. In different measuring times, sEMG signals during MVC task were measured for five times, and the five-point moving average of the sEMG signals is used for normalization of sEMG signals, which is measured in simulated driving experiments.
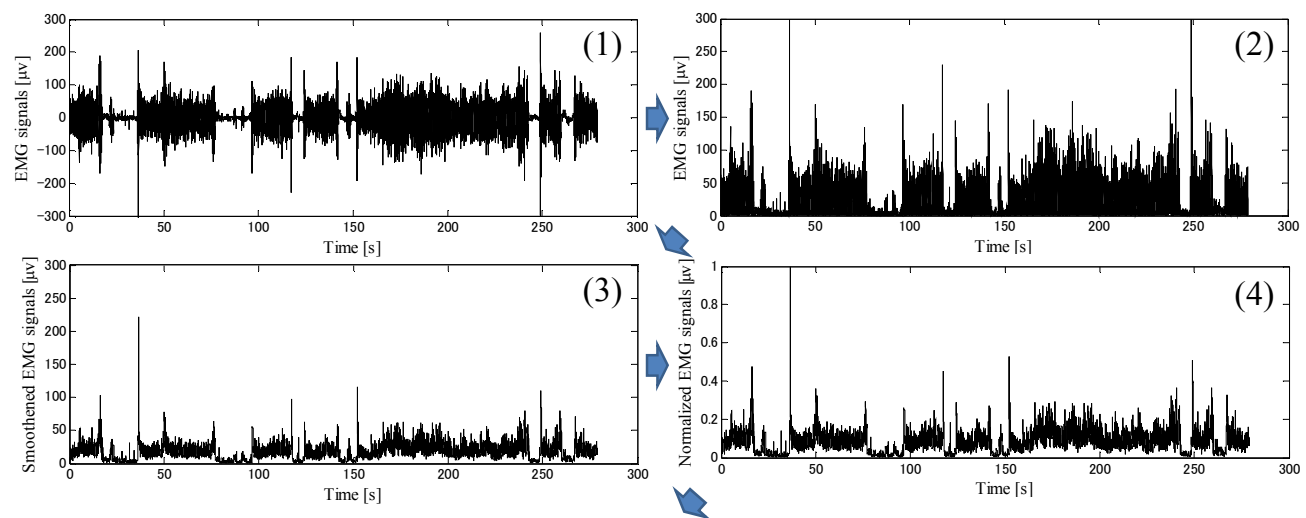

(5) RMS level

Fig. 5 Signal processing of sEMG

\section{Results and Discussions}

By 10 subjects driving experiments, fuel economies in three driving modes were calculated. The fuel economy of normal driving is $9.86 \pm 1.84 \mathrm{~km} / 1$ (Mean \pm standard deviation), fuel economy of eco-driving with instruction $11.96 \pm 0.63 \mathrm{~km} / \mathrm{l}$, and fuel economy of eco-driving with eco-indicator $12.41 \pm 0.36 \mathrm{~km} / 1$, respectively. The results show that two eco-driving modes are apparently efficient to improve fuel consumption. Especially, eco-driving with eco-indicator is optimal driving mode for fuel economy. Sequent, Based on the results of fuel economy, physical condition of driver would be discussed through measured sEMG signals and proposed processing method.

\subsection{Muscular activities}

To analyze the physical condition of leg, a representative result of sEMG of tibialis anterior muscle is presented in Fig. 6. The figure shows sEMG signals in the first period [I] and second period [II] by the modes of normal driving, eco-driving, and eco-driving with eco-indicator. In Fig. 6, X-axis is a time scale [s], and Y-axis is electrical voltage scale [ $\mu \mathrm{v}]$. The line of $30 \mu \mathrm{v}$ is added as an indication of amplitude of sEMG signal. sEMG signals around $0 \mu \mathrm{v}$ represents the interval stopping with red signal by subjects. The sampling frequency is $250 \mathrm{~Hz}$, and the shift integral time is for one second. 

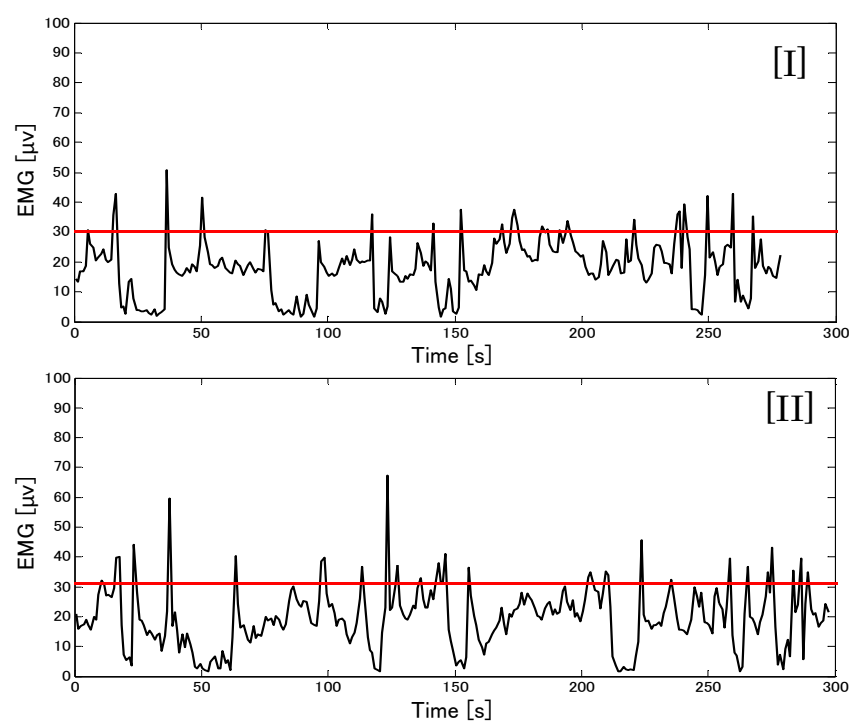

(a) Normal driving
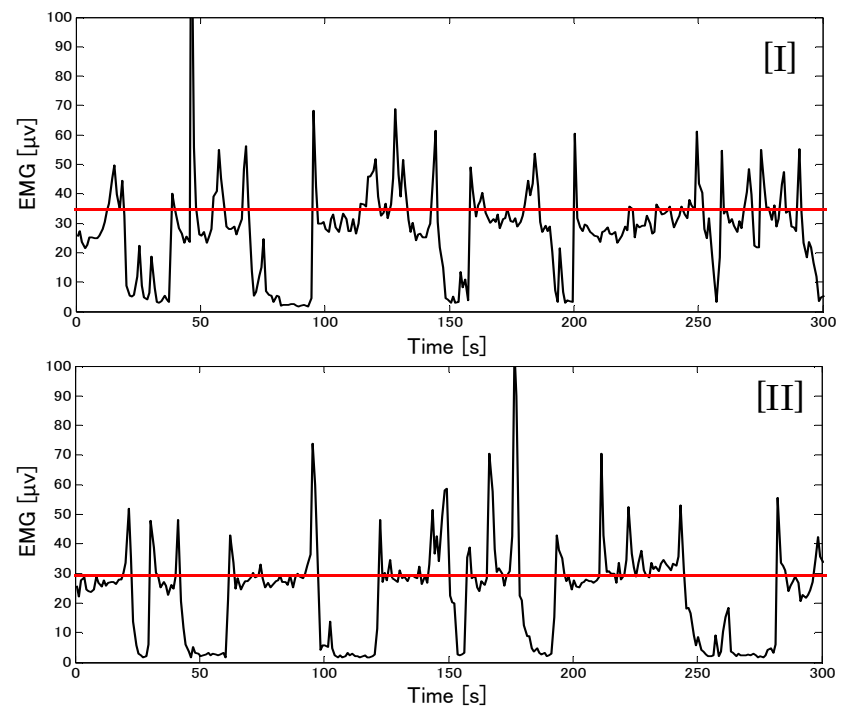

(b) Eco-driving with instruction
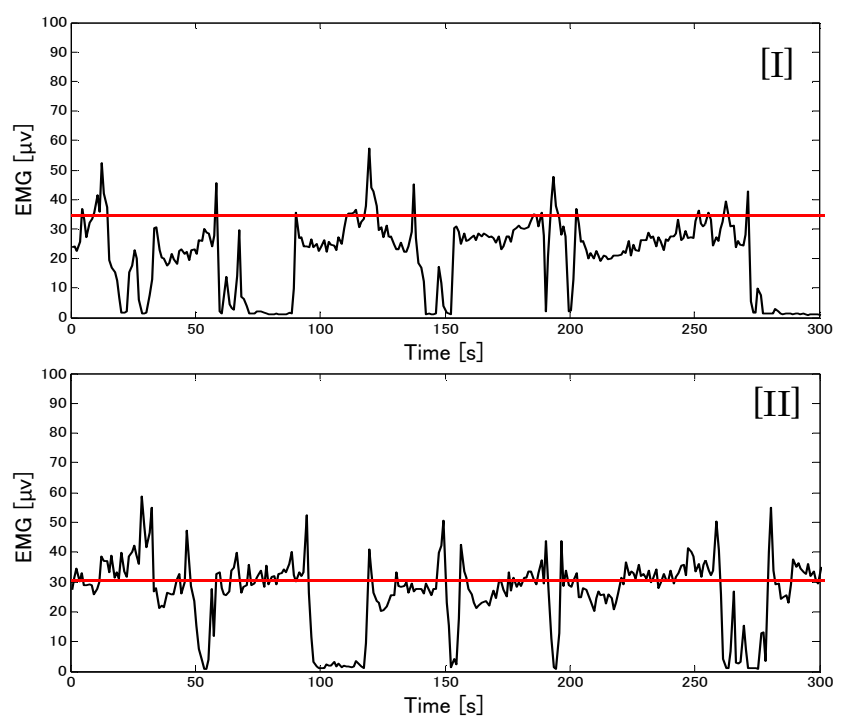

(c) Eco-driving with eco-indicator

Fig. 6 sEMG results by shift integral processing 
By comparison sEMG signals of [I] and [II] in Fig. 6 (a), the signals after departure time becomes overactive and over $30 \mu \mathrm{v}$ line, and sEMG signals in [II] of Fig. 6 (a) show that the muscle of leg is being tired relative to sEMG signals in [I] of Fig. 6 (a). On the other hand, sEMG signals aren't clearly different between [I] and [II] in Fig. 6 (b). By observing sEMG signals in normal driving of Fig. 6 (a), the driver uses the muscular strength clearly to keep gently accelerating and braking, even the peaks of sEMG are also large.

sEMG signals are not much different between in Fig. 6 (a) and (c), however, sEMG signals in Fig. 6 (c) have less times over $30 \mu \mathrm{v}$ than in Fig. 6 (b). Additionally, the average of sEMG in eco-driving with instruction and with eco-indicator is about $30 \mu \mathrm{v}$, but the average of sEMG in normal driving is about $20 \mu \mathrm{v}$. A parentally difference is observed before stopping, between Fig. 6 (b) and (c), which is a movement of early accelerator off of eco-driving with indicator, but eco-driving with instruction did extreme operations. The reason is that present mileage could not be understood by subject, which resulted of using extra muscular strength. The result indicates the driver performs accelerator off with the understanding of the current mileage when an eco-indicator is added, which is the effectiveness of the eco-indicator as an eco-assistant system.

\subsection{Muscular fatigue}

By driving experiment of 10 subjects, sEMG signals of tibialis anterior muscle were measured, and results of RMS values after the proposed signal processing are shown in Fig. 7.



(a) Mean values

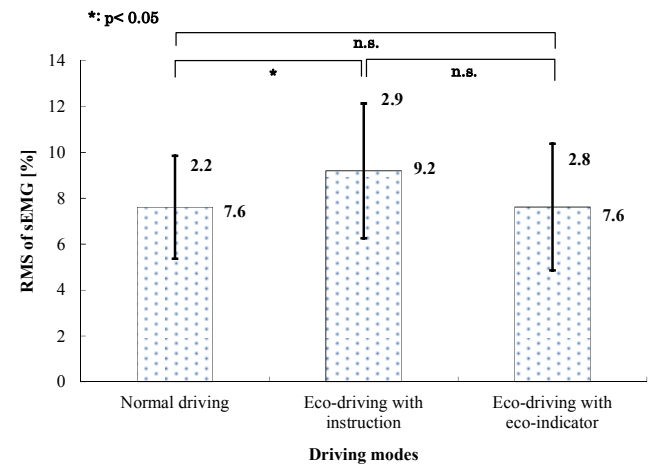

(b) First period



(c) Second period

Fig. 7 RMS values of sEMG 
In the Fig. 7, the RMS values are presented by mean values and standard deviations. After calculating RMS values of sMEG signals in the first period and the second period of traffic scenario (in Section 2.3), results presented in Fig. 7(a) are mean values of sum of the RMS values of the two periods. The RMS value of normal driving is $7.5 \pm 2.0$, the RMS value of eco-driving with instruction is $9.3 \pm 3.0$, and RMS value of eco-driving with eco-indicator $7.3 \pm 2.4$. When the RMS value is used as a valuation index of muscular fatigue, the subject in the mode of eco-driving with instruction is most getting muscular fatigue of leg. Reversely, the mode of eco-driving with eco-indicator could improve muscular fatigue of the subject, even though the two modes are effective to improve the fuel economy.

The results in Fig. 7 (b) are RMS values during the first period of the traffic scenario. The RMS value of normal driving is $7.6 \pm 2.2$, the RMS value of eco-driving with instruction is $9.2 \pm 2.9$, and the RMS value of eco-driving with eco-indicator $7.6 \pm 2.8$. The results in Fig. 7 (c) are RMS values of second period of traffic scenario. RMS value of normal driving is $7.4 \pm 2.1$, RMS value of eco-driving with instruction is $9.5 \pm 3.3$, and RMS value of eco-driving with eco-indicator $7.0 \pm 3.1$.

From the results in Figs 7 (b) and 7 (c), the same conclusion could be drawn comparing with the results in Fig. 7 (a), which is that eco-driving with eco-indicator is helpful to decrease muscular fatigue of the subject. The distinct point, between the results in Figs 7 (b) and 7 (c) about the RMS values of eco-driving with eco-indicator, is that the tendency becomes more obvious after 30 minutes driving.

The total amount of sEMG signals in one lap is shown in Fig. 8. In the Fig. 8, the results are presented by mean values and standard deviations of 10 subjects. For analysis of the main tendencies, mean values of total amount $s$ of sEMG in first period and second period of traffic scenario are presented in Fig. 8 (a). The total values of sEMG of normal driving is $1599 \pm 462$, the total value of sEMG of eco-driving with instruction $2228 \pm 652$, and the total value of sEMG of eco-driving with eco-indicator $2069 \pm 444$, respectively. By above results, muscular strength of leg with eco-driving modes is more required than normal driving mode. The reason is that driving time increases for one circle in the traffic scenario in the case of eco-driving mode. In details, the mean driving time is $318 \mathrm{~s}$ for normal driving, however, the mean driving time for eco-driving with instructions is $354 \mathrm{~s}$, and eco-driving with eco-indicator is 368 s. The gentle operations of acceleration and brake by eco-driving modes effect driving velocities.

The total values of sEMG during first period of traffic scenario are presented in Fig. 8 (b). The total value of sEMG of normal driving is $1610 \pm 410$, the total value of sEMG of eco-driving with instruction $2213 \pm 450$, and the total value of eco-driving with eco-indicator $2184 \pm 675$, respectively. The total values of sEMG of second period of traffic scenario are presented in Fig. 8 (c). The total value of normal driving is $1589 \pm 592$, the total value of eco-driving with instruction $22412 \pm 924$, and the total value of eco-driving with eco-indicator $1953 \pm 615$, respectively. Comparing the results in Fig. 8 (b) and 8 (c), the total amount of sEMG in eco-driving with indicator apparently decreased, but there are not obviously different with the mode of eco-driving with instruction.

In a word, comparing the results of eco-driving with instruction and eco-driving with eco-indicator modes, muscular fatigue of leg could be improved by eco-indicator. By the way, eco-driving experiments for each driving mode were completed over 30 minutes, and executed in different days. The arrangement of the experiment reduces the effects of experimental sequence. 


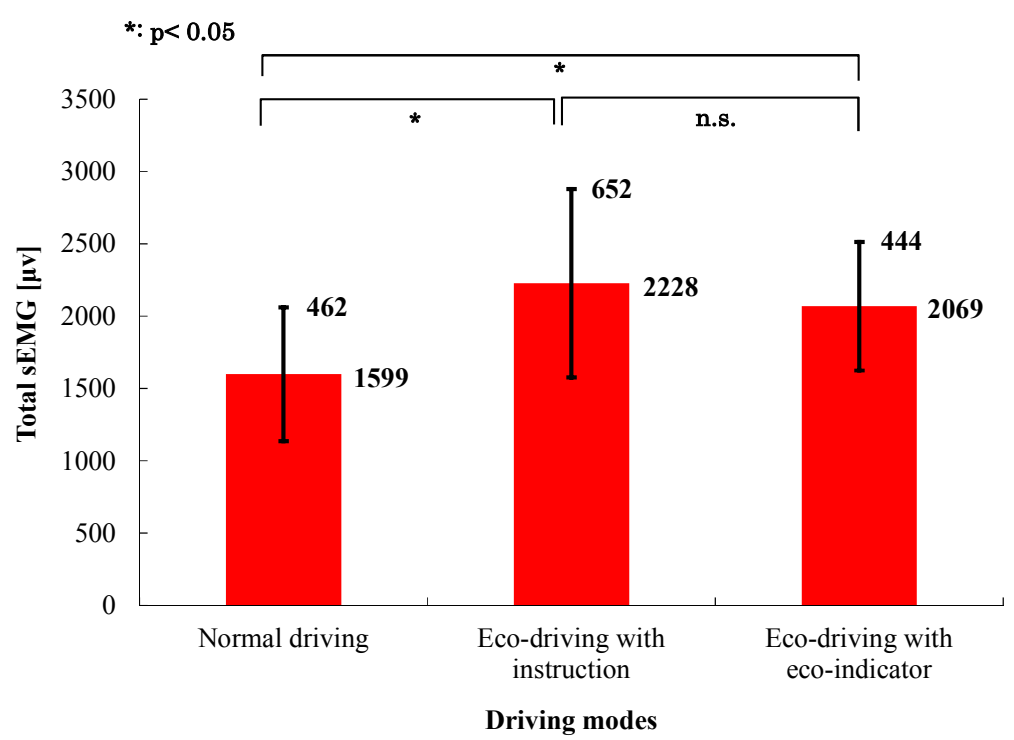

(a) Mean values

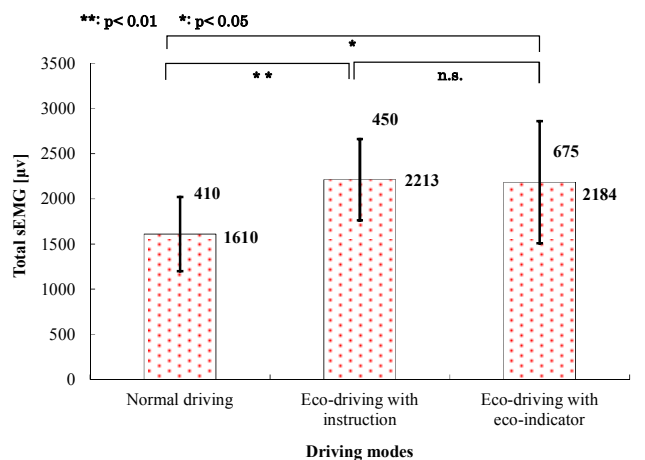

(b) First period

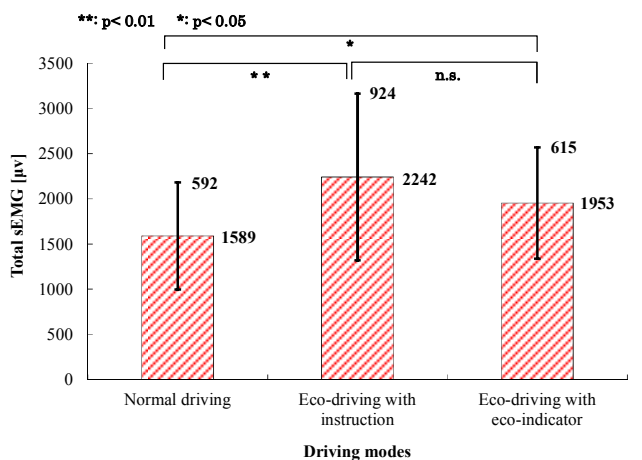

(c) Second period

Fig. 8 Total amount of sEMG

\section{Conclusions}

This paper presents experimental studies about eco-driving behaviors by a universal driving simulator. Muscular activities and fatigues in the modes of normal driving, eco-driving with instruction, and eco-driving with eco-indicator were analyzed for further understand driver behaviors in eco-driving. By the studies, several conclusions were drawn as followings:

In our experiment, sEMG signal of maximum voluntary contraction (MVC) is used to normalize sEMG data, and the results of muscle activities and fatigues were estimated.

(1) The results suggested that muscle activities of driver became more sensitive to the movement of stop and departure with the traffic signal. Moreover, driver should use the leg muscular strength gently to keep driving with a little acceleration and deceleration for reduction muscle fatigue.

(2) By comparing the results of total amounts of sEMG, driving time increases in eco-driving mode, and resulted that more muscular strength were used to realize eco-driving. But, in the mode of eco-driving with eco-indicator, driving time decreased, and physical fatigue of driver is improve relative to eco-driving mode.

(3) Muscle fatigue of driver in normal driving, eco-driving with instruction, and eco-driving with eco-indicator were analyzed, and the studied results indicated that eco-driving with eco-indicator is effective driving mode on fuel consumption reduction as well as on reduce physical burden of driver. 


\section{Acknowledgement}

The research was conducted as a part of Energy-ITS Project, and financially supported by New Energy and Industrial Technology Development Organization of Japan (NEDO). Moreover, authors gratefully acknowledge Dr. Toshihiro Hiraoka in Kyoto University and Dr. Shuichi Matsumoto in Keio University for their constructive suggestions to the eco-driving experiment study.

\section{References}

(1) Masaaki Taniguchi, A Study On Eco-driving and Energy Saving, Global Warming Prevention with ITS, (2008), Society of Automotive Engineer of Japan Inc.

(2) John Conquest, Ian Patey, Aidan Holt, Using ITS to Cut Carbon Costs, 15th World Congress on Intelligent Transport Systems, (2008).

(3) Toshihiro Hiraoka, Yasuhiro Terakado, Shuichi Matsumoto, Shigeyuki Yamabe, Quantitative Evaluation of Eco-driving on Fuel Consumption based on Driving Simulator Experiments, 16th World Congress on Intelligent Transport Systems, (2009).

(4) Ryosuke Ando, Yasuhide Nishihori, Daisuke Ochi, Development of a System to Promote Eco-Driving and Safe-Driving, Smart Spaces and Next Generation Wired/Wireless networking, (2010), Vol. 6294, pp. 207-218, DOI: 10.1007/978-3-642-14891-0_19

(5) Woodrow Barfield, Thomas A. Dingus, Human Factor in Intelligent Transportation System, (1997), Lawrence Erlbaum Associates.

(6) Team-6\%committee \& Ministry of the Environment, (2011) http://www.team-6.jp/ecodrive/10recommendation/index.html

(7) Daisuke Yamaguchi, Yoshihiro Suda, Masaaki Onuki, Toshihiko Oda, Hiroki Ishikawa and Hong Ilgi, Improvement of Realistic Sensation on Universal Driving Simulator, Proceedings of the 21st International Symposium on Dynamics of Vehicles on Roads and Tracks, (2009).

(8) Matthew Barth, et al., Development of a Comprehensive Modal Emission Model, (2000), National Cooperative Highway Research Program.

(9) Mieko Ohsuga, Ethical Issues Related to the Experiments using Human Subjects, JSAE Annual Congress of Spring, (2009).

(10) Kizuka Tomohiro, Masuda Tadashi, Kiryu Tohru, Sadoyama Tsugutake, Biomechanical Library Practical Usage of Surface Electromyogram, Tokyo Denki University Press, (2008).

(11) Aldo O. Perotto, Anatomical Guide for the Electromyographer: the Limbs and Truck, (2005), Charles C Thomas.

(12) R. T. Floyd, Manual of Structural Kinesiology, (2006), McGraw-Hill.

(13) O. C. J. Lippold, J. W. T. Redfearn, J. Vuco, The Electromyography of Fatigue, Ergonomics, Vol. 3, No. 2, (1960), pp.121-131.

(14) I. Hostens, H. Ramon, Assessment of Muscle Fatigue in Low Level Monotonous Task Performance during Car Driving, Journal of Electromyography and Kinesiology, Vol. 15, No. 3, (2005), pp.266-274. 